Acta Crystallographica Section E

Structure Reports

Online

ISSN 1600-5368

\section{(Dibenzo-18-crown-6)(2-phenylamidopyridine)- potassium(I)}

The title complex, $\left[\mathrm{Rb}\left(\mathrm{C}_{11} \mathrm{H}_{9} \mathrm{~N}_{2}\right)\left(\mathrm{C}_{20} \mathrm{H}_{24} \mathrm{O}_{6}\right)\right]$, has potassium in an irregular eightfold coordination, with a hexadentate crown ether ligand and a chelating bidentate amidopyridine ligand, each occupying one hemisphere of the coordination. The chelate KNCN ring is slightly folded, and the two rings of the amidopyridine ligand are not coplanar, because of steric interaction of $\mathrm{H}$ atoms on the rings. The $\mathrm{K}-\mathrm{N}$ (amido) bond is shorter than the $\mathrm{K}-\mathrm{N}$ (pyridine) bond. The coordination of the crown ether to potassium is less symmetrical than that for the analogous rubidium complex, reflecting a poorer size match for $\mathrm{K}^{+}$in this coordination site.

\section{Comment}

In the preceding paper (Liddle \& Clegg, 2004), we described the structure of the complex (dibenzo-18-crown-6) $\mathrm{Rb}(L)$, where $\mathrm{H} L$ is 2-phenylaminopyridine. We present here the structure of the analogous potassium complex, (dibenzo-18crown-6)K(L), (I). These complexes were prepared as part of a study of crown-ether-supported complexes of alkali metals with amide ligands, and were investigated specifically for comparison with the complexes with 18-crown-6 (Liddle et al., 2004; Liddle \& Clegg, 2003).

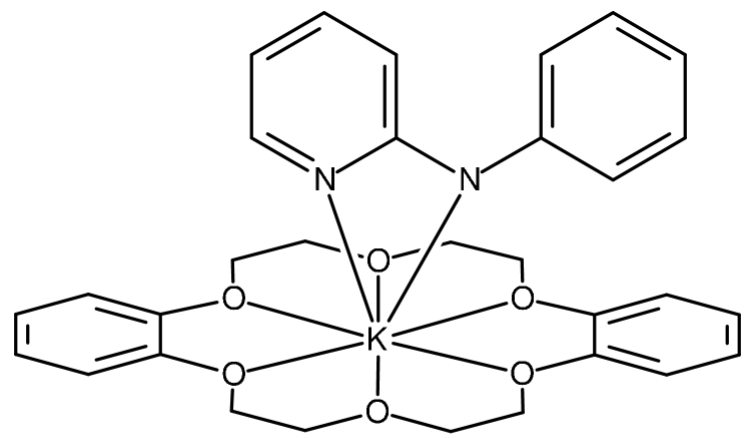

(I)
Received 14 September 2004 Accepted 20 September 2004 Online 30 September 2004
For details of how these key indicators were automatically derived from the article, see http://journals.iucr.org/e.
(C) 2004 International Union of Crystallography Printed in Great Britain - all rights reserved
Although the $\mathrm{Rb}$ complexes with the two different crown ethers are structurally very similar, there is a marked difference for the $\mathrm{K}$ complexes. Reaction of equimolar amounts of 18-crown-6, potassium hydride and $\mathrm{H} L$ leads to the polymeric complex $\left[\left(18 \text {-crown-6) } \mathrm{K}(L)_{2} \mathrm{~K}\right]_{\infty}\right.$, even though this product has a 1:2:2 stoichiometry (Liddle et al., 2004). With dibenzo-18crown-6 instead of 18-crown-6, the title complex (with a 1:1:1 stoichiometry) is obtained, exactly analogous to the $\mathrm{Rb}$ case.

The dibenzo-18-crown- 6 complexes of $\mathrm{Rb}$ and $\mathrm{K}$ are structurally similar in gross terms; there are subtle but significant differences in detail. Fig. 1 shows the molecular structure of the title $\mathrm{K}$ complex, and selected geometric 
parameters are in Table 1 . It consists of discrete neutral molecules with no special intermolecular interactions. The hexadentate crown ligand and the bidentate amide anion occupy the two coordination hemispheres of the potassium ion, giving irregular eightfold coordination, and the complex may be described as a contact ion pair.

The range of $\mathrm{K}-\mathrm{O}$ distances and the difference between the two $\mathrm{K}-\mathrm{N}$ distances are both somewhat greater than the corresponding values for the $\mathrm{Rb}$ complex, and this probably reflects the poorer fit of the smaller potassium ion in the coordination site; it is generally recognized that 18 -crown- 6 and its substituted derivatives provide an ideal fit for $\mathrm{K}^{+}$in the mean plane of the six $\mathrm{O}$ atoms rather than displaced from this plane. In the title complex, K lies 0.7830 (7) $\AA$ out of the oxygen mean plane (r.m.s. deviation $0.009 \AA$ ), compared with a deviation of 1.0945 (6) $\AA$ for $\mathrm{Rb}$ in the analogous complex. Although all four $\mathrm{O}-\mathrm{C}-\mathrm{C}-\mathrm{O}$ aliphatic segments have a gauche conformation, as is expected for optimal chelation, the overall conformation of the crown in this complex differs from that in the $\mathrm{Rb}$ complex by conversion of one anti $\mathrm{C}-\mathrm{C}-\mathrm{O}-$ $\mathrm{C}$ linkage to gauche, the other eleven remaining anti (Table 1). The crown ligand is thus rather less symmetrical in the title complex than in the $\mathrm{Rb}$ complex. The two benzene rings are folded out of the oxygen mean plane, away from the amide ligand by $39.97(6)$ and $8.52(7)^{\circ}$, in contrast to the angles of $22.93(7)$ and $25.94(7)^{\circ}$ towards the amide ligand in the $\mathrm{Rb}$ complex.

The two rings of the amide ligand have a dihedral angle of $49.91(7)^{\circ}$ because of steric interaction of $\mathrm{H}$ atoms bonded to $\mathrm{C} 4$ and $\mathrm{C} 11$. The four-membered chelate ring (KNCN) is approximately planar, the dihedral angle between the $\mathrm{KN}_{2}$ and $\mathrm{CN}_{2}$ planes being $7.3(2)^{\circ}$, compared with only $0.3(3)^{\circ}$ in the $\mathrm{Rb}$ complex, once again displaying the effects of greater geometrical strain from the size mismatch of the metal ion and its ligand set.

\section{Experimental}

Potassium hydride $(0.04 \mathrm{~g}, 1.0 \mathrm{mmol})$ was added to a solution of 2-phenylaminopyridine $(0.17 \mathrm{~g}, 1.0 \mathrm{mmol})$ and dibenzo-18-crown-6 $(0.36 \mathrm{~g}, 1.0 \mathrm{mmol})$ in tetrahydrofuran (THF, $40 \mathrm{ml})$, to give a pale yellow precipitate. Volatile components were removed in vacuo and the remaining solid was washed with petroleum ether $(3 \times 5 \mathrm{ml})$. Recrystallization from hot toluene containing a little hexamethylphosphoramide (HMPA) gave yellow crystals of (I) (yield $0.38 \mathrm{~g}$, $67 \%$ ). Chemical analysis results were satisfactory, and the ${ }^{1} \mathrm{H}$ and ${ }^{13} \mathrm{C}\left\{{ }^{1} \mathrm{H}\right\}$ NMR signals could be assigned on the basis of the crystal structure (Liddle, 2000).

\section{Crystal data}

$\left[\mathrm{K}\left(\mathrm{C}_{11} \mathrm{H}_{9} \mathrm{~N}_{2}\right)\left(\mathrm{C}_{20} \mathrm{H}_{24} \mathrm{O}_{6}\right)\right]$

$M_{r}=568.69$

Monoclinic, $C 2 / c$

$a=26.950(2) \AA$

$b=10.3120(9) \AA$

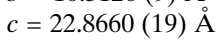

$\beta=117.607$ (2)

$V=5631.0(8) \AA^{3}$

$Z=8$

\author{
$D_{x}=1.342 \mathrm{Mg} \mathrm{m}^{-3}$ \\ Mo $K \alpha$ radiation \\ Cell parameters from 7626 \\ reflections \\ $\theta=2.5-27.8^{\circ}$ \\ $\mu=0.24 \mathrm{~mm}^{-1}$ \\ $T=160$ (2) K \\ Needle, yellow \\ $0.82 \times 0.12 \times 0.10 \mathrm{~mm}$
}

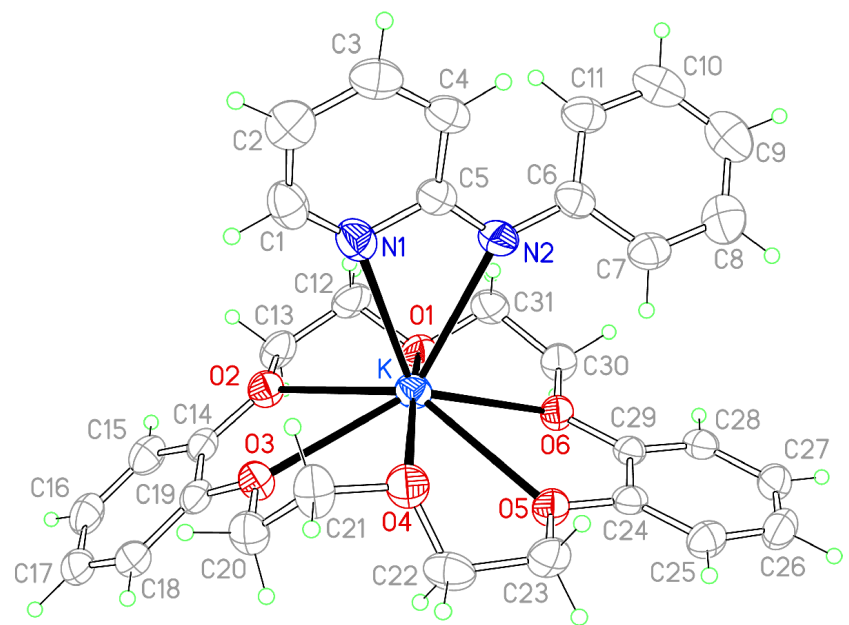

Figure 1

The molecular structure of (I), showing the atom labels and $50 \%$ probability displacement ellipsoids for non-H atoms.

Data collection

Bruker SMART 1K CCD diffractometer

Thin-slice $\omega$ scans

Absorption correction: multi-scan (SADABS; Sheldrick, 2002)

$T_{\min }=0.830, T_{\max }=0.977$

21735 measured reflections

Refinement

Refinement on $F^{2}$

$R\left[F^{2}>2 \sigma\left(F^{2}\right)\right]=0.043$

$w R\left(F^{2}\right)=0.110$

$S=1.04$

6673 reflections

361 parameters

$\mathrm{H}$-atom parameters constrained

6673 independent reflections 4349 reflections with $I>2 \sigma(I)$

$R_{\text {int }}=0.037$

$\theta_{\max }=28.6^{\circ}$

$h=-35 \rightarrow 35$

$k=-13 \rightarrow 12$

$l=-30 \rightarrow 29$

Table 1

Selected geometric parameters $\left(\AA{ }^{\circ}\right)$.

\begin{tabular}{lclc}
\hline $\mathrm{K}-\mathrm{N} 1$ & $2.8515(18)$ & $\mathrm{K}-\mathrm{O} 5$ & $2.8374(12)$ \\
$\mathrm{K}-\mathrm{N} 2$ & $2.7935(16)$ & $\mathrm{K}-\mathrm{O} 6$ & $2.8194(12)$ \\
$\mathrm{K}-\mathrm{O} 1$ & $2.8156(13)$ & $\mathrm{N} 1-\mathrm{C} 1$ & $1.331(3)$ \\
$\mathrm{K}-\mathrm{O} 2$ & $2.8578(12)$ & $\mathrm{N} 1-\mathrm{C} 5$ & $1.368(2)$ \\
$\mathrm{K}-\mathrm{O} 3$ & $2.8519(12)$ & $\mathrm{N} 2-\mathrm{C} 5$ & $1.340(2)$ \\
$\mathrm{K}-\mathrm{O} 4$ & $2.7138(13)$ & $\mathrm{N} 2-\mathrm{C} 6$ & $1.384(2)$ \\
& & & \\
$\mathrm{N} 1-\mathrm{K}-\mathrm{N} 2$ & $47.72(5)$ & $\mathrm{K}-\mathrm{N} 1-\mathrm{C} 1$ & $143.34(14)$ \\
$\mathrm{O} 1-\mathrm{K}-\mathrm{O} 2$ & $60.36(4)$ & $\mathrm{K}-\mathrm{N} 1-\mathrm{C} 5$ & $96.70(12)$ \\
$\mathrm{O} 1-\mathrm{K}-\mathrm{O} 6$ & $58.97(3)$ & $\mathrm{C} 1-\mathrm{N} 1-\mathrm{C} 5$ & $118.40(18)$ \\
$\mathrm{O} 2-\mathrm{K}-\mathrm{O} 3$ & $54.13(3)$ & $\mathrm{K}-\mathrm{N} 2-\mathrm{C} 5$ & $100.10(11)$ \\
$\mathrm{O} 3-\mathrm{K}-\mathrm{O} 4$ & $61.85(4)$ & $\mathrm{K}-\mathrm{N} 2-\mathrm{C} 6$ & $123.11(16)$ \\
$\mathrm{O} 4-\mathrm{K}-\mathrm{O} 5$ & $60.48(4)$ & $\mathrm{C} 5-\mathrm{N} 2-\mathrm{C} 6$ & \\
$\mathrm{O} 5-\mathrm{K}-\mathrm{O} 6$ & $54.89(3)$ & $\mathrm{N} 1-\mathrm{C} 5-\mathrm{N} 2$ & \\
& & & $-67.4(2)$ \\
$\mathrm{C} 6-\mathrm{N} 2-\mathrm{C} 5-\mathrm{N} 1$ & $168.74(17)$ & $\mathrm{C} 22-\mathrm{O} 4-\mathrm{C} 21-\mathrm{C} 20$ & $-17.0(2)$ \\
$\mathrm{C} 6-\mathrm{N} 2-\mathrm{C} 5-\mathrm{C} 4$ & $-16.0(3)$ & $\mathrm{O} 3-\mathrm{C} 20-\mathrm{C} 21-\mathrm{O} 4$ & -58.0 \\
$\mathrm{C} 5-\mathrm{N} 2-\mathrm{C} 6-\mathrm{C} 7$ & $145.31(18)$ & $\mathrm{C} 21-\mathrm{O} 4-\mathrm{C} 22-\mathrm{C} 23$ & $-170.92(14)$ \\
$\mathrm{C} 5-\mathrm{N} 2-\mathrm{C} 6-\mathrm{C} 11$ & $-40.6(3)$ & $\mathrm{C} 24-\mathrm{O} 5-\mathrm{C} 23-\mathrm{C} 22$ & $-171.65(15)$ \\
$\mathrm{C} 31-\mathrm{O} 1-\mathrm{C} 12-\mathrm{C} 13$ & $-179.51(15)$ & $\mathrm{O} 4-\mathrm{C} 22-\mathrm{C} 23-\mathrm{O} 5$ & $-62.1(2)$ \\
$\mathrm{C} 14-\mathrm{O} 2-\mathrm{C} 13-\mathrm{C} 12$ & $-174.33(15)$ & $\mathrm{C} 23-\mathrm{O} 5-\mathrm{C} 24-\mathrm{C} 29$ & $-175.53(16)$ \\
$\mathrm{O} 1-\mathrm{C} 12-\mathrm{C} 13-\mathrm{O} 2$ & $68.2(2)$ & $\mathrm{C} 30-\mathrm{O} 6-\mathrm{C} 29-\mathrm{C} 24$ & $175.55(16)$ \\
$\mathrm{C} 13-\mathrm{O} 2-\mathrm{C} 14-\mathrm{C} 19$ & $170.87(15)$ & $\mathrm{O} 5-\mathrm{C} 24-\mathrm{C} 29-\mathrm{O} 6$ & $3.8(2)$ \\
$\mathrm{C} 20-\mathrm{O} 3-\mathrm{C} 19-\mathrm{C} 14$ & $175.63(15)$ & $\mathrm{C} 29-\mathrm{O} 6-\mathrm{C} 30-\mathrm{C} 31$ & $-158.91(15)$ \\
$\mathrm{O} 2-\mathrm{C} 14-\mathrm{C} 19-\mathrm{O} 3$ & $2.3(2)$ & $\mathrm{C} 12-\mathrm{O} 1-\mathrm{C} 31-\mathrm{C} 30$ & $-179.30(15)$ \\
$\mathrm{C} 19-\mathrm{O} 3-\mathrm{C} 20-\mathrm{C} 21$ & $-168.71(16)$ & $\mathrm{O} 6-\mathrm{C} 30-\mathrm{C} 31-\mathrm{O} 1$ & $-59.1(2)$ \\
\hline & & &
\end{tabular}


$\mathrm{H}$ atoms were positioned geometrically, with $\mathrm{C}-\mathrm{H}=0.95$ (aromatic) or $0.99 \AA$ (aliphatic), and refined with a riding model, with $U_{\text {iso }}(\mathrm{H})=1.2 U_{\text {eq }}(\mathrm{C})$.

Data collection: SMART (Bruker, 2001); cell refinement: local programs; data reduction: SAINT (Bruker, 2001); program(s) used to solve structure: SHELXTL (Sheldrick, 2001); program(s) used to refine structure: SHELXTL; molecular graphics: SHELXTL; software used to prepare material for publication: SHELXTL and local programs.
We thank the EPSRC for financial support.

\section{References}

Bruker (2001). SMART and SAINT. Bruker AXS Inc., Madison, Wisconsin, USA.

Liddle, S. T. (2000). PhD thesis, University of Newcastle upon Tyne, England. Liddle, S. T. \& Clegg, W. (2003). Polyhedron, 22, 3507-3513.

Liddle, S. T. \& Clegg, W. (2004). Acta Cryst. E60, m1492-m1494.

Liddle, S. T., Clegg, W. \& Morrison, C. A. (2004). Dalton Trans. pp. 2514-2525.

Sheldrick, G. M. (2001). SHELXTL. Version 5. Bruker AXS Inc., Madison, Wisconsin, USA.

Sheldrick, G. M. (2002). SADABS. University of Göttingen, Germany. 


\section{supporting information}

Acta Cryst. (2004). E60, m1495-m1497 [https://doi.org/10.1107/S1600536804023360]

\section{(Dibenzo-18-crown-6)(2-phenylamidopyridine)potassium(I)}

\section{William Clegg and Stephen T. Liddle}

(Dibenzo-18-crown-6)(2-phenylamidopyridine)potassium(I)

Crystal data

$\left[\mathrm{K}\left(\mathrm{C}_{11} \mathrm{H}_{9} \mathrm{~N}_{2}\right)\left(\mathrm{C}_{20} \mathrm{H}_{24} \mathrm{O}_{6}\right)\right]$

$M_{r}=568.69$

Monoclinic, $C 2 / c$

$a=26.950(2) \AA$

$b=10.3120(9) \AA$

$c=22.8660(19) \AA$

$\beta=117.607(2)^{\circ}$

$V=5631.0(8) \AA^{3}$

$Z=8$

\section{Data collection}

Bruker SMART 1K CCD diffractometer

Radiation source: sealed tube

Graphite monochromator

Detector resolution: 8.192 pixels $\mathrm{mm}^{-1}$

thin-slice $\omega$ scans

Absorption correction: multi-scan

(SADABS; Sheldrick, 2002)

$T_{\min }=0.830, T_{\max }=0.977$

\section{Refinement}

Refinement on $F^{2}$

Least-squares matrix: full

$R\left[F^{2}>2 \sigma\left(F^{2}\right)\right]=0.043$

$w R\left(F^{2}\right)=0.110$

$S=1.04$

6673 reflections

361 parameters

0 restraints

Primary atom site location: structure-invariant direct methods
$F(000)=2400$

$D_{\mathrm{x}}=1.342 \mathrm{Mg} \mathrm{m}^{-3}$

Mo $K \alpha$ radiation, $\lambda=0.71073 \AA$

Cell parameters from 7626 reflections

$\theta=2.5-27.8^{\circ}$

$\mu=0.24 \mathrm{~mm}^{-1}$

$T=160 \mathrm{~K}$

Needle, yellow

$0.82 \times 0.12 \times 0.10 \mathrm{~mm}$

21735 measured reflections

6673 independent reflections

4349 reflections with $I>2 \sigma(I)$

$R_{\text {int }}=0.037$

$\theta_{\max }=28.6^{\circ}, \theta_{\min }=1.7^{\circ}$

$h=-35 \rightarrow 35$

$k=-13 \rightarrow 12$

$l=-30 \rightarrow 29$

Secondary atom site location: difference Fourier map

Hydrogen site location: inferred from neighbouring sites

$\mathrm{H}$-atom parameters constrained

$w=1 /\left[\sigma^{2}\left(F_{\mathrm{o}}^{2}\right)+(0.0525 P)^{2}+1.0053 P\right]$

where $P=\left(F_{\mathrm{o}}^{2}+2 F_{\mathrm{c}}{ }^{2}\right) / 3$

$(\Delta / \sigma)_{\max }<0.001$

$\Delta \rho_{\max }=0.77 \mathrm{e} \AA^{-3}$

$\Delta \rho_{\min }=-0.35$ e $\AA^{-3}$

Fractional atomic coordinates and isotropic or equivalent isotropic displacement parameters $\left(\AA^{2}\right)$

\begin{tabular}{lllll}
\hline & $x$ & $y$ & $z$ & $U_{\text {iso }} * U_{\text {eq }}$ \\
\hline $\mathrm{K}$ & $0.147063(16)$ & $0.53950(4)$ & $0.039410(19)$ & $0.03171(12)$ \\
$\mathrm{N} 1$ & $0.10174(7)$ & $0.75945(17)$ & $-0.04426(8)$ & $0.0419(4)$ \\
$\mathrm{N} 2$ & $0.10649(7)$ & $0.77716(16)$ & $0.05777(8)$ & $0.0403(4)$
\end{tabular}




\begin{tabular}{|c|c|c|c|c|}
\hline $\mathrm{C} 1$ & $0.08226(8)$ & $0.8005(2)$ & $-0.10619(10)$ & $0.0439(5)$ \\
\hline $\mathrm{H} 1 \mathrm{~A}$ & 0.0922 & 0.7509 & -0.1342 & $0.053^{*}$ \\
\hline $\mathrm{C} 2$ & $0.04928(9)$ & $0.9074(2)$ & $-0.13350(11)$ & $0.0484(5)$ \\
\hline $\mathrm{H} 2 \mathrm{~A}$ & 0.0370 & 0.9316 & -0.1782 & $0.058^{*}$ \\
\hline C3 & $0.03478(8)$ & $0.9785(2)$ & $-0.09186(10)$ & $0.0440(5)$ \\
\hline $\mathrm{H} 3 \mathrm{~A}$ & 0.0111 & 1.0520 & -0.1083 & $0.053 *$ \\
\hline $\mathrm{C} 4$ & $0.05458(8)$ & $0.94262(19)$ & $-0.02746(10)$ & $0.0371(5)$ \\
\hline $\mathrm{H} 4 \mathrm{~A}$ & 0.0457 & 0.9935 & 0.0011 & $0.045^{*}$ \\
\hline $\mathrm{C} 5$ & $0.08864(7)$ & $0.82902(19)$ & $-0.00237(10)$ & $0.0338(4)$ \\
\hline C6 & $0.10561(7)$ & $0.84335(19)$ & $0.10993(9)$ & 0.0359 (4) \\
\hline $\mathrm{C} 7$ & $0.09463(8)$ & $0.7730(2)$ & $0.15545(10)$ & $0.0396(5)$ \\
\hline H7A & 0.0857 & 0.6834 & 0.1477 & $0.048 *$ \\
\hline $\mathrm{C} 8$ & $0.09632(9)$ & $0.8296(2)$ & $0.21069(10)$ & $0.0507(6)$ \\
\hline $\mathrm{H} 8 \mathrm{~A}$ & 0.0882 & 0.7790 & 0.2399 & $0.061 *$ \\
\hline C9 & $0.10969(9)$ & $0.9589(3)$ & $0.22430(11)$ & $0.0555(6)$ \\
\hline H9A & 0.1106 & 0.9980 & 0.2624 & $0.067 *$ \\
\hline $\mathrm{C} 10$ & $0.12162(8)$ & $1.0302(2)$ & $0.18134(11)$ & $0.0498(6)$ \\
\hline $\mathrm{H} 10 \mathrm{~A}$ & 0.1314 & 1.1191 & 0.1904 & $0.060 *$ \\
\hline C11 & $0.11964(8)$ & $0.9746(2)$ & $0.12542(11)$ & $0.0421(5)$ \\
\hline H11A & 0.1279 & 1.0263 & 0.0967 & $0.051 *$ \\
\hline $\mathrm{O} 1$ & $0.25742(5)$ & $0.59682(12)$ & $0.13446(6)$ & $0.0334(3)$ \\
\hline $\mathrm{O} 2$ & $0.23595(5)$ & $0.52324(13)$ & $0.00460(6)$ & $0.0345(3)$ \\
\hline $\mathrm{O} 3$ & $0.14500(5)$ & $0.39465(12)$ & $-0.06794(6)$ & $0.0329(3)$ \\
\hline $\mathrm{O} 4$ & $0.05117(5)$ & $0.41353(14)$ & $-0.04173(6)$ & $0.0413(3)$ \\
\hline $\mathrm{O} 5$ & $0.09313(5)$ & $0.38149(12)$ & $0.09450(6)$ & $0.0330(3)$ \\
\hline O6 & $0.18057(5)$ & $0.51384(12)$ & $0.17537(6)$ & $0.0328(3)$ \\
\hline $\mathrm{C} 12$ & $0.27351(9)$ & $0.6723(2)$ & $0.09390(10)$ & $0.0421(5)$ \\
\hline $\mathrm{H} 12 \mathrm{~A}$ & 0.3068 & 0.7252 & 0.1218 & $0.051 *$ \\
\hline H12B & 0.2427 & 0.7317 & 0.0661 & $0.051^{*}$ \\
\hline $\mathrm{C} 13$ & $0.28664(8)$ & $0.5838(2)$ & $0.05116(9)$ & $0.0398(5)$ \\
\hline $\mathrm{H} 13 \mathrm{~A}$ & 0.3033 & 0.6337 & 0.0277 & $0.048 *$ \\
\hline H13B & 0.3139 & 0.5171 & 0.0786 & $0.048^{*}$ \\
\hline $\mathrm{C} 14$ & $0.24077(8)$ & $0.42932(18)$ & $-0.03513(9)$ & $0.0311(4)$ \\
\hline $\mathrm{C} 15$ & $0.28920(8)$ & $0.4022(2)$ & $-0.03978(10)$ & $0.0409(5)$ \\
\hline H15A & 0.3222 & 0.4512 & -0.0148 & $0.049 *$ \\
\hline C16 & $0.28944(9)$ & $0.3031(2)$ & $-0.08100(10)$ & $0.0432(5)$ \\
\hline H16A & 0.3228 & 0.2848 & -0.0839 & $0.052 *$ \\
\hline C17 & $0.24219(9)$ & $0.2317(2)$ & $-0.11733(10)$ & $0.0409(5)$ \\
\hline H17A & 0.2428 & 0.1635 & -0.1449 & $0.049 *$ \\
\hline $\mathrm{C} 18$ & $0.19340(8)$ & $0.25955(18)$ & $-0.11352(9)$ & $0.0360(4)$ \\
\hline $\mathrm{H} 18 \mathrm{~A}$ & 0.1605 & 0.2104 & -0.1388 & $0.043 *$ \\
\hline C19 & $0.19227(7)$ & $0.35806(18)$ & $-0.07329(8)$ & $0.0301(4)$ \\
\hline $\mathrm{C} 20$ & $0.09366(8)$ & $0.3299(2)$ & $-0.10991(9)$ & $0.0391(5)$ \\
\hline $\mathrm{H} 20 \mathrm{~A}$ & 0.0949 & 0.2391 & -0.0952 & $0.047^{*}$ \\
\hline H20B & 0.0879 & 0.3290 & -0.1559 & $0.047^{*}$ \\
\hline $\mathrm{C} 21$ & $0.04672(8)$ & $0.4021(2)$ & $-0.10602(9)$ & $0.0440(5)$ \\
\hline $\mathrm{H} 21 \mathrm{~A}$ & 0.0447 & 0.4904 & -0.1240 & $0.053^{*}$ \\
\hline H21B & 0.0111 & 0.3578 & -0.1348 & $0.053^{*}$ \\
\hline
\end{tabular}




$\begin{array}{lllll}\mathrm{C} 22 & 0.04578(9) & 0.2961(2) & -0.01491(9) & 0.0436(5) \\ \mathrm{H} 22 \mathrm{~A} & 0.0786 & 0.2404 & -0.0053 & 0.052^{*} \\ \mathrm{H} 22 \mathrm{~B} & 0.0118 & 0.2502 & -0.0471 & 0.052^{*} \\ \mathrm{C} 23 & 0.04172(8) & 0.3205(2) & 0.04790(9) & 0.0379(5) \\ \mathrm{H} 23 \mathrm{~A} & 0.0095 & 0.3778 & 0.0389 & 0.046^{*} \\ \mathrm{H} 23 \mathrm{~B} & 0.0363 & 0.2377 & 0.0661 & 0.046^{*} \\ \mathrm{C} 24 & 0.10019(7) & 0.39557(17) & 0.15784(9) & 0.0285(4) \\ \mathrm{C} 25 & 0.06357(8) & 0.34800(19) & 0.17935(9) & 0.0359(4) \\ \mathrm{H} 25 \mathrm{~A} & 0.0306 & 0.3037 & 0.1495 & 0.043^{*} \\ \mathrm{C} 26 & 0.07504(8) & 0.3650(2) & 0.24480(10) & 0.0413(5) \\ \mathrm{H} 26 \mathrm{~A} & 0.0496 & 0.3328 & 0.2593 & 0.050^{*} \\ \mathrm{C} 27 & 0.12269(8) & 0.42753(19) & 0.28840(10) & 0.0381(5) \\ \mathrm{H} 27 \mathrm{~A} & 0.1304 & 0.4374 & 0.3331 & 0.046^{*} \\ \mathrm{C} 28 & 0.15986(8) & 0.47670(18) & 0.26747(9) & 0.0325(4) \\ \mathrm{H} 28 \mathrm{~A} & 0.1931 & 0.5193 & 0.2980 & 0.039^{*} \\ \mathrm{C} 29 & 0.14839(7) & 0.46343(17) & 0.20197(9) & 0.0279(4) \\ \mathrm{C} 30 & 0.22798(8) & 0.5921(2) & 0.21820(9) & 0.0378(5) \\ \mathrm{H} 30 \mathrm{~A} & 0.2598 & 0.5357 & 0.2466 & 0.045^{*} \\ \mathrm{H} 30 \mathrm{~B} & 0.2181 & 0.6465 & 0.2470 & 0.045^{*} \\ \mathrm{C} 31 & 0.24386(8) & 0.67620(19) & 0.17617(9) & 0.0378(5) \\ \mathrm{H} 31 \mathrm{~A} & 0.2123 & 0.7344 & 0.1489 & 0.045^{*} \\ \mathrm{H} 31 \mathrm{~B} & 0.2765 & 0.7305 & 0.2046 & 0.045^{*}\end{array}$

Atomic displacement parameters $\left(\AA^{2}\right)$

\begin{tabular}{lllllll}
\hline & $U^{11}$ & $U^{22}$ & $U^{33}$ & $U^{12}$ & $U^{13}$ & $U^{23}$ \\
\hline $\mathrm{K}$ & $0.0283(2)$ & $0.0301(2)$ & $0.0363(2)$ & $-0.00319(17)$ & $0.01459(17)$ & $-0.00488(18)$ \\
$\mathrm{N} 1$ & $0.0407(10)$ & $0.0413(10)$ & $0.0480(10)$ & $0.0019(8)$ & $0.0241(9)$ & $-0.0050(8)$ \\
$\mathrm{N} 2$ & $0.0455(10)$ & $0.0303(10)$ & $0.0514(10)$ & $0.0059(7)$ & $0.0279(9)$ & $0.0009(8)$ \\
$\mathrm{C} 1$ & $0.0385(11)$ & $0.0501(14)$ & $0.0480(13)$ & $-0.0072(10)$ & $0.0243(10)$ & $-0.0132(11)$ \\
$\mathrm{C} 2$ & $0.0422(12)$ & $0.0519(14)$ & $0.0437(12)$ & $-0.0096(11)$ & $0.0135(10)$ & $-0.0036(11)$ \\
$\mathrm{C} 3$ & $0.0354(11)$ & $0.0343(12)$ & $0.0539(13)$ & $-0.0046(9)$ & $0.0135(10)$ & $-0.0034(10)$ \\
$\mathrm{C} 4$ & $0.0305(10)$ & $0.0306(11)$ & $0.0504(12)$ & $-0.0021(8)$ & $0.0188(9)$ & $-0.0054(9)$ \\
$\mathrm{C} 5$ & $0.0262(9)$ & $0.0307(11)$ & $0.0483(12)$ & $-0.0057(8)$ & $0.0204(9)$ & $-0.0050(9)$ \\
$\mathrm{C} 6$ & $0.0282(10)$ & $0.0346(11)$ & $0.0429(11)$ & $0.0063(8)$ & $0.0148(9)$ & $-0.0002(9)$ \\
$\mathrm{C} 7$ & $0.0377(11)$ & $0.0347(12)$ & $0.0436(12)$ & $0.0062(9)$ & $0.0164(9)$ & $0.0066(9)$ \\
$\mathrm{C} 8$ & $0.0455(12)$ & $0.0655(17)$ & $0.0328(11)$ & $0.0113(11)$ & $0.0111(10)$ & $0.0089(11)$ \\
$\mathrm{C} 9$ & $0.0502(14)$ & $0.0667(18)$ & $0.0345(12)$ & $0.0122(12)$ & $0.0068(10)$ & $-0.0092(12)$ \\
$\mathrm{C} 10$ & $0.0359(11)$ & $0.0429(13)$ & $0.0528(13)$ & $0.0041(10)$ & $0.0055(10)$ & $-0.0121(11)$ \\
$\mathrm{C} 11$ & $0.0356(11)$ & $0.0341(12)$ & $0.0557(13)$ & $-0.0012(9)$ & $0.0203(10)$ & $-0.0023(10)$ \\
O1 & $0.0344(7)$ & $0.0303(7)$ & $0.0372(7)$ & $-0.0076(5)$ & $0.0181(6)$ & $-0.0014(6)$ \\
O2 & $0.0305(7)$ & $0.0371(8)$ & $0.0377(7)$ & $-0.0063(6)$ & $0.0172(6)$ & $-0.0032(6)$ \\
O3 & $0.0281(7)$ & $0.0366(8)$ & $0.0343(7)$ & $-0.0029(5)$ & $0.0148(6)$ & $-0.0058(6)$ \\
O4 & $0.0387(8)$ & $0.0444(9)$ & $0.0412(8)$ & $-0.0078(6)$ & $0.0188(6)$ & $-0.0066(6)$ \\
O5 & $0.0297(7)$ & $0.0365(8)$ & $0.0325(7)$ & $-0.0114(5)$ & $0.0140(6)$ & $-0.0072(6)$ \\
O6 & $0.0319(7)$ & $0.0353(8)$ & $0.0336(7)$ & $-0.0122(5)$ & $0.0171(6)$ & $-0.0089(6)$ \\
C12 & $0.0436(11)$ & $0.0375(12)$ & $0.0457(12)$ & $-0.0190(9)$ & $0.0211(10)$ & $-0.0029(10)$ \\
C13 & $0.0349(11)$ & $0.0456(13)$ & $0.0403(11)$ & $-0.0150(9)$ & $0.0185(9)$ & $-0.0003(9)$ \\
& & & & & &
\end{tabular}




$\begin{array}{lllllll}\text { C14 } & 0.0335(10) & 0.0326(11) & 0.0313(10) & 0.0018(8) & 0.0184(8) & 0.0070(8) \\ \text { C15 } & 0.0361(11) & 0.0466(13) & 0.0452(11) & -0.0001(9) & 0.0233(9) & 0.0085(10) \\ \text { C16 } & 0.0453(12) & 0.0474(13) & 0.0497(12) & 0.0135(10) & 0.0329(11) & 0.0152(10) \\ \text { C17 } & 0.0574(14) & 0.0332(12) & 0.0423(11) & 0.0107(10) & 0.0317(11) & 0.0088(9) \\ \text { C18 } & 0.0425(11) & 0.0332(11) & 0.0346(10) & 0.0025(9) & 0.0199(9) & 0.0040(9) \\ \text { C19 } & 0.0334(10) & 0.0309(10) & 0.0291(9) & 0.0035(8) & 0.0169(8) & 0.0084(8) \\ \text { C20 } & 0.0347(10) & 0.0451(13) & 0.0372(11) & -0.0098(9) & 0.0164(9) & -0.0099(9) \\ \text { C21 } & 0.0304(10) & 0.0640(15) & 0.0354(11) & -0.0074(10) & 0.0133(9) & -0.0094(10) \\ \text { C22 } & 0.0432(12) & 0.0334(12) & 0.0397(11) & -0.0067(9) & 0.0071(9) & -0.0085(9) \\ \text { C23 } & 0.0307(10) & 0.0394(12) & 0.0400(11) & -0.0130(8) & 0.0131(9) & -0.0057(9) \\ \text { C24 } & 0.0286(9) & 0.0210(10) & 0.0364(10) & 0.0022(7) & 0.0156(8) & 0.0017(8) \\ \text { C25 } & 0.0297(10) & 0.0359(11) & 0.0414(11) & -0.0034(8) & 0.0160(9) & 0.0017(9) \\ \text { C26 } & 0.0400(11) & 0.0441(13) & 0.0484(12) & 0.0020(9) & 0.0278(10) & 0.0097(10) \\ \text { C27 } & 0.0468(12) & 0.0364(12) & 0.0361(11) & 0.0047(9) & 0.0235(10) & 0.0037(9) \\ \text { C28 } & 0.0362(10) & 0.0257(10) & 0.0349(10) & 0.0013(8) & 0.0160(8) & -0.0002(8) \\ \text { C29 } & 0.0289(9) & 0.0216(9) & 0.0351(10) & 0.0010(7) & 0.0165(8) & -0.0001(8) \\ \text { C30 } & 0.0315(10) & 0.0428(12) & 0.0373(11) & -0.0145(9) & 0.0143(9) & -0.0121(9) \\ \text { C31 } & 0.0343(10) & 0.0336(11) & 0.0435(11) & -0.0113(8) & 0.0164(9) & -0.0108(9)\end{array}$

Geometric parameters $\left(A,{ }^{\circ}\right)$

\begin{tabular}{llll}
\hline $\mathrm{K}-\mathrm{N} 1$ & $2.8515(18)$ & $\mathrm{O} 6-\mathrm{C} 30$ & $1.443(2)$ \\
$\mathrm{K}-\mathrm{N} 2$ & $2.7935(16)$ & $\mathrm{C} 12-\mathrm{H} 12 \mathrm{~A}$ & 0.990 \\
$\mathrm{~K}-\mathrm{O} 1$ & $2.8156(13)$ & $\mathrm{C} 12-\mathrm{H} 12 \mathrm{~B}$ & 0.990 \\
$\mathrm{~K}-\mathrm{O} 2$ & $2.8578(12)$ & $\mathrm{C} 12-\mathrm{C} 13$ & $1.495(3)$ \\
$\mathrm{K}-\mathrm{O} 3$ & $2.8519(12)$ & $\mathrm{C} 13-\mathrm{H} 13 \mathrm{~A}$ & 0.990 \\
$\mathrm{~K}-\mathrm{O} 4$ & $2.7138(13)$ & $\mathrm{C} 13-\mathrm{H} 13 \mathrm{~B}$ & 0.990 \\
$\mathrm{~K}-\mathrm{O} 5$ & $2.8374(12)$ & $\mathrm{C} 14-\mathrm{C} 15$ & $1.386(3)$ \\
$\mathrm{K}-\mathrm{O} 6$ & $2.8194(12)$ & $\mathrm{C} 14-\mathrm{C} 19$ & $1.398(3)$ \\
$\mathrm{N} 1-\mathrm{C} 1$ & $1.331(3)$ & $\mathrm{C} 15-\mathrm{H} 15 \mathrm{~A}$ & 0.950 \\
$\mathrm{~N} 1-\mathrm{C} 5$ & $1.368(2)$ & $\mathrm{C} 15-\mathrm{C} 16$ & $1.392(3)$ \\
$\mathrm{N} 2-\mathrm{C} 5$ & $1.340(2)$ & $\mathrm{C} 16-\mathrm{H} 16 \mathrm{~A}$ & 0.950 \\
$\mathrm{~N} 2-\mathrm{C} 6$ & $1.384(2)$ & $\mathrm{C} 16-\mathrm{C} 17$ & $1.369(3)$ \\
$\mathrm{C} 1-\mathrm{H} 1 \mathrm{~A}$ & 0.950 & $\mathrm{C} 17-\mathrm{H} 17 \mathrm{~A}$ & 0.950 \\
$\mathrm{C} 1-\mathrm{C} 2$ & $1.372(3)$ & $\mathrm{C} 17-\mathrm{C} 18$ & $1.387(3)$ \\
$\mathrm{C} 2-\mathrm{H} 2 \mathrm{~A}$ & 0.950 & $\mathrm{C} 18-\mathrm{H} 18 \mathrm{~A}$ & 0.950 \\
$\mathrm{C} 2-\mathrm{C} 3$ & $1.393(3)$ & $\mathrm{C} 18-\mathrm{C} 19$ & $1.380(3)$ \\
$\mathrm{C} 3-\mathrm{H} 3 \mathrm{~A}$ & 0.950 & $\mathrm{C} 20-\mathrm{H} 20 \mathrm{~A}$ & 0.990 \\
$\mathrm{C} 3-\mathrm{C} 4$ & $1.364(3)$ & $\mathrm{C} 20-\mathrm{H} 20 \mathrm{~B}$ & 0.990 \\
$\mathrm{C} 4-\mathrm{H} 4 \mathrm{~A}$ & 0.950 & $\mathrm{C} 20-\mathrm{C} 21$ & $1.506(3)$ \\
$\mathrm{C} 4-\mathrm{C} 5$ & $1.434(3)$ & $\mathrm{C} 21-\mathrm{H} 21 \mathrm{~A}$ & 0.990 \\
$\mathrm{C} 6-\mathrm{C} 7$ & $1.408(3)$ & $\mathrm{C} 21-\mathrm{H} 21 \mathrm{~B}$ & 0.990 \\
$\mathrm{C} 6-\mathrm{C} 11$ & $1.406(3)$ & $\mathrm{C} 22-\mathrm{H} 22 \mathrm{~A}$ & 0.990 \\
$\mathrm{C} 7-\mathrm{H} 7 \mathrm{~A}$ & 0.950 & $\mathrm{C} 22-\mathrm{H} 22 \mathrm{~B}$ & 0.990 \\
$\mathrm{C} 7-\mathrm{C} 8$ & $1.373(3)$ & $\mathrm{C} 22-\mathrm{C} 23$ & $1.511(3)$ \\
$\mathrm{C} 8-\mathrm{H} 8 \mathrm{~A}$ & 0.950 & $\mathrm{C} 23-\mathrm{H} 23 \mathrm{~A}$ & 0.990 \\
$\mathrm{C} 8-\mathrm{C} 9$ & $1.379(3)$ & $\mathrm{C} 23-\mathrm{H} 23 \mathrm{~B}$ & 0.990 \\
$\mathrm{C} 9-\mathrm{H} 9 \mathrm{~A}$ & 0.950 & $\mathrm{C} 24-\mathrm{C} 25$ & $1.381(2)$ \\
& & &
\end{tabular}




\begin{tabular}{|c|c|c|c|}
\hline $\mathrm{C} 9-\mathrm{C} 10$ & $1.379(3)$ & $\mathrm{C} 24-\mathrm{C} 29$ & $1.407(2)$ \\
\hline $\mathrm{C} 10-\mathrm{H} 10 \mathrm{~A}$ & 0.950 & $\mathrm{C} 25-\mathrm{H} 25 \mathrm{~A}$ & 0.950 \\
\hline $\mathrm{C} 10-\mathrm{C} 11$ & $1.380(3)$ & $\mathrm{C} 25-\mathrm{C} 26$ & $1.392(3)$ \\
\hline $\mathrm{C} 11-\mathrm{H} 11 \mathrm{~A}$ & 0.950 & $\mathrm{C} 26-\mathrm{H} 26 \mathrm{~A}$ & 0.950 \\
\hline $\mathrm{O} 1-\mathrm{C} 12$ & $1.424(2)$ & $\mathrm{C} 26-\mathrm{C} 27$ & $1.368(3)$ \\
\hline $\mathrm{O} 1-\mathrm{C} 31$ & $1.427(2)$ & $\mathrm{C} 27-\mathrm{H} 27 \mathrm{~A}$ & 0.950 \\
\hline $\mathrm{O} 2-\mathrm{C} 13$ & $1.429(2)$ & $\mathrm{C} 27-\mathrm{C} 28$ & $1.391(3)$ \\
\hline $\mathrm{O} 2-\mathrm{C} 14$ & $1.375(2)$ & $\mathrm{C} 28-\mathrm{H} 28 \mathrm{~A}$ & 0.950 \\
\hline $\mathrm{O} 3-\mathrm{C} 19$ & $1.387(2)$ & $\mathrm{C} 28-\mathrm{C} 29$ & $1.389(2)$ \\
\hline $\mathrm{O} 3-\mathrm{C} 20$ & $1.432(2)$ & $\mathrm{C} 30-\mathrm{H} 30 \mathrm{~A}$ & 0.990 \\
\hline $\mathrm{O} 4-\mathrm{C} 21$ & $1.423(2)$ & $\mathrm{C} 30-\mathrm{H} 30 \mathrm{~B}$ & 0.990 \\
\hline $\mathrm{O} 4-\mathrm{C} 22$ & $1.395(2)$ & $\mathrm{C} 30-\mathrm{C} 31$ & $1.497(3)$ \\
\hline $\mathrm{O} 5-\mathrm{C} 23$ & $1.443(2)$ & $\mathrm{C} 31-\mathrm{H} 31 \mathrm{~A}$ & 0.990 \\
\hline $\mathrm{O} 5-\mathrm{C} 24$ & $1.378(2)$ & $\mathrm{C} 31-\mathrm{H} 31 \mathrm{~B}$ & 0.990 \\
\hline $\mathrm{O} 6-\mathrm{C} 29$ & $1.372(2)$ & & \\
\hline $\mathrm{N} 1-\mathrm{K}-\mathrm{N} 2$ & $47.72(5)$ & $\mathrm{O} 1-\mathrm{C} 12-\mathrm{H} 12 \mathrm{~A}$ & 109.9 \\
\hline $\mathrm{N} 1-\mathrm{K}-\mathrm{O} 1$ & $108.77(4)$ & $\mathrm{O} 1-\mathrm{C} 12-\mathrm{H} 12 \mathrm{~B}$ & 109.9 \\
\hline $\mathrm{N} 1-\mathrm{K}-\mathrm{O} 2$ & $90.77(4)$ & $\mathrm{O} 1-\mathrm{C} 12-\mathrm{C} 13$ & $109.13(16)$ \\
\hline $\mathrm{N} 1-\mathrm{K}-\mathrm{O} 3$ & $90.44(4)$ & $\mathrm{H} 12 \mathrm{~A}-\mathrm{C} 12-\mathrm{H} 12 \mathrm{~B}$ & 108.3 \\
\hline $\mathrm{N} 1-\mathrm{K}-\mathrm{O} 4$ & $86.90(5)$ & $\mathrm{H} 12 \mathrm{~A}-\mathrm{C} 12-\mathrm{C} 13$ & 109.9 \\
\hline $\mathrm{N} 1-\mathrm{K}-\mathrm{O} 5$ & $127.32(4)$ & $\mathrm{H} 12 \mathrm{~B}-\mathrm{C} 12-\mathrm{C} 13$ & 109.9 \\
\hline $\mathrm{N} 1-\mathrm{K}-\mathrm{O} 6$ & $129.29(4)$ & $\mathrm{O} 2-\mathrm{C} 13-\mathrm{C} 12$ & $108.66(15)$ \\
\hline $\mathrm{N} 2-\mathrm{K}-\mathrm{O} 1$ & $92.68(4)$ & $\mathrm{O} 2-\mathrm{C} 13-\mathrm{H} 13 \mathrm{~A}$ & 110.0 \\
\hline $\mathrm{N} 2-\mathrm{K}-\mathrm{O} 2$ & $122.03(4)$ & $\mathrm{O} 2-\mathrm{C} 13-\mathrm{H} 13 \mathrm{~B}$ & 110.0 \\
\hline $\mathrm{N} 2-\mathrm{K}-\mathrm{O} 3$ & $137.39(5)$ & $\mathrm{C} 12-\mathrm{C} 13-\mathrm{H} 13 \mathrm{~A}$ & 110.0 \\
\hline $\mathrm{N} 2-\mathrm{K}-\mathrm{O} 4$ & $101.98(5)$ & $\mathrm{C} 12-\mathrm{C} 13-\mathrm{H} 13 \mathrm{~B}$ & 110.0 \\
\hline $\mathrm{N} 2-\mathrm{K}-\mathrm{O} 5$ & $96.91(4)$ & $\mathrm{H} 13 \mathrm{~A}-\mathrm{C} 13-\mathrm{H} 13 \mathrm{~B}$ & 108.3 \\
\hline $\mathrm{N} 2-\mathrm{K}-\mathrm{O} 6$ & $82.20(4)$ & $\mathrm{O} 2-\mathrm{C} 14-\mathrm{C} 15$ & $125.02(17)$ \\
\hline $\mathrm{O} 1-\mathrm{K}-\mathrm{O} 2$ & $60.36(4)$ & $\mathrm{O} 2-\mathrm{C} 14-\mathrm{C} 19$ & $115.82(15)$ \\
\hline $\mathrm{O} 1-\mathrm{K}-\mathrm{O} 3$ & $111.51(4)$ & $\mathrm{C} 15-\mathrm{C} 14-\mathrm{C} 19$ & $119.16(18)$ \\
\hline $\mathrm{O} 1-\mathrm{K}-\mathrm{O} 4$ & $163.51(4)$ & $\mathrm{C} 14-\mathrm{C} 15-\mathrm{H} 15 \mathrm{~A}$ & 120.0 \\
\hline $\mathrm{O} 1-\mathrm{K}-\mathrm{O} 5$ & $110.67(4)$ & $\mathrm{C} 14-\mathrm{C} 15-\mathrm{C} 16$ & $120.02(19)$ \\
\hline $\mathrm{O} 1-\mathrm{K}-\mathrm{O} 6$ & $58.97(3)$ & $\mathrm{H} 15 \mathrm{~A}-\mathrm{C} 15-\mathrm{C} 16$ & 120.0 \\
\hline $\mathrm{O} 2-\mathrm{K}-\mathrm{O} 3$ & $54.13(3)$ & $\mathrm{C} 15-\mathrm{C} 16-\mathrm{H} 16 \mathrm{~A}$ & 119.6 \\
\hline $\mathrm{O} 2-\mathrm{K}-\mathrm{O} 4$ & $115.92(4)$ & $\mathrm{C} 15-\mathrm{C} 16-\mathrm{C} 17$ & $120.71(18)$ \\
\hline $\mathrm{O} 2-\mathrm{K}-\mathrm{O} 5$ & $139.28(4)$ & $\mathrm{H} 16 \mathrm{~A}-\mathrm{C} 16-\mathrm{C} 17$ & 119.6 \\
\hline $\mathrm{O} 2-\mathrm{K}-\mathrm{O} 6$ & $114.84(4)$ & $\mathrm{C} 16-\mathrm{C} 17-\mathrm{H} 17 \mathrm{~A}$ & 120.2 \\
\hline $\mathrm{O} 3-\mathrm{K}-\mathrm{O} 4$ & $61.85(4)$ & $\mathrm{C} 16-\mathrm{C} 17-\mathrm{C} 18$ & $119.5(2)$ \\
\hline $\mathrm{O} 3-\mathrm{K}-\mathrm{O} 5$ & $105.76(4)$ & $\mathrm{H} 17 \mathrm{~A}-\mathrm{C} 17-\mathrm{C} 18$ & 120.2 \\
\hline $\mathrm{O} 3-\mathrm{K}-\mathrm{O} 6$ & $140.22(4)$ & $\mathrm{C} 17-\mathrm{C} 18-\mathrm{H} 18 \mathrm{~A}$ & 119.7 \\
\hline $\mathrm{O} 4-\mathrm{K}-\mathrm{O} 5$ & $60.48(4)$ & $\mathrm{C} 17-\mathrm{C} 18-\mathrm{C} 19$ & $120.58(19)$ \\
\hline $\mathrm{O} 4-\mathrm{K}-\mathrm{O} 6$ & $115.21(4)$ & $\mathrm{H} 18 \mathrm{~A}-\mathrm{C} 18-\mathrm{C} 19$ & 119.7 \\
\hline $\mathrm{O} 5-\mathrm{K}-\mathrm{O} 6$ & $54.89(3)$ & $\mathrm{O} 3-\mathrm{C} 19-\mathrm{C} 14$ & $115.69(16)$ \\
\hline $\mathrm{K}-\mathrm{N} 1-\mathrm{C} 1$ & $143.34(14)$ & $\mathrm{O} 3-\mathrm{C} 19-\mathrm{C} 18$ & $124.32(17)$ \\
\hline $\mathrm{K}-\mathrm{N} 1-\mathrm{C} 5$ & $96.70(12)$ & $\mathrm{C} 14-\mathrm{C} 19-\mathrm{C} 18$ & $119.99(17)$ \\
\hline $\mathrm{C} 1-\mathrm{N} 1-\mathrm{C} 5$ & $118.40(18)$ & $\mathrm{O} 3-\mathrm{C} 20-\mathrm{H} 20 \mathrm{~A}$ & 110.1 \\
\hline $\mathrm{K}-\mathrm{N} 2-\mathrm{C} 5$ & $100.10(11)$ & $\mathrm{O} 3-\mathrm{C} 20-\mathrm{H} 20 \mathrm{~B}$ & 110.1 \\
\hline
\end{tabular}




\begin{tabular}{|c|c|c|c|}
\hline $\mathrm{K}-\mathrm{N} 2-\mathrm{C} 6$ & $136.59(12)$ & $\mathrm{O} 3-\mathrm{C} 20-\mathrm{C} 21$ & $107.87(15)$ \\
\hline $\mathrm{C} 5-\mathrm{N} 2-\mathrm{C} 6$ & $123.11(16)$ & $\mathrm{H} 20 \mathrm{~A}-\mathrm{C} 20-\mathrm{H} 20 \mathrm{~B}$ & 108.4 \\
\hline $\mathrm{N} 1-\mathrm{C} 1-\mathrm{H} 1 \mathrm{~A}$ & 116.8 & $\mathrm{H} 20 \mathrm{~A}-\mathrm{C} 20-\mathrm{C} 21$ & 110.1 \\
\hline $\mathrm{N} 1-\mathrm{C} 1-\mathrm{C} 2$ & $126.4(2)$ & $\mathrm{H} 20 \mathrm{~B}-\mathrm{C} 20-\mathrm{C} 21$ & 110.1 \\
\hline $\mathrm{H} 1 \mathrm{~A}-\mathrm{C} 1-\mathrm{C} 2$ & 116.8 & $\mathrm{O} 4-\mathrm{C} 21-\mathrm{C} 20$ & $115.52(17)$ \\
\hline $\mathrm{C} 1-\mathrm{C} 2-\mathrm{H} 2 \mathrm{~A}$ & 122.0 & $\mathrm{O} 4-\mathrm{C} 21-\mathrm{H} 21 \mathrm{~A}$ & 108.4 \\
\hline $\mathrm{C} 1-\mathrm{C} 2-\mathrm{C} 3$ & $116.1(2)$ & $\mathrm{O} 4-\mathrm{C} 21-\mathrm{H} 21 \mathrm{~B}$ & 108.4 \\
\hline $\mathrm{H} 2 \mathrm{~A}-\mathrm{C} 2-\mathrm{C} 3$ & 122.0 & $\mathrm{C} 20-\mathrm{C} 21-\mathrm{H} 21 \mathrm{~A}$ & 108.4 \\
\hline $\mathrm{C} 2-\mathrm{C} 3-\mathrm{H} 3 \mathrm{~A}$ & 119.9 & $\mathrm{C} 20-\mathrm{C} 21-\mathrm{H} 21 \mathrm{~B}$ & 108.4 \\
\hline $\mathrm{C} 2-\mathrm{C} 3-\mathrm{C} 4$ & $120.1(2)$ & $\mathrm{H} 21 \mathrm{~A}-\mathrm{C} 21-\mathrm{H} 21 \mathrm{~B}$ & 107.5 \\
\hline $\mathrm{H} 3 \mathrm{~A}-\mathrm{C} 3-\mathrm{C} 4$ & 119.9 & $\mathrm{O} 4-\mathrm{C} 22-\mathrm{H} 22 \mathrm{~A}$ & 109.7 \\
\hline $\mathrm{C} 3-\mathrm{C} 4-\mathrm{H} 4 \mathrm{~A}$ & 119.6 & $\mathrm{O} 4-\mathrm{C} 22-\mathrm{H} 22 \mathrm{~B}$ & 109.7 \\
\hline $\mathrm{C} 3-\mathrm{C} 4-\mathrm{C} 5$ & $120.73(19)$ & $\mathrm{O} 4-\mathrm{C} 22-\mathrm{C} 23$ & $109.93(16)$ \\
\hline $\mathrm{H} 4 \mathrm{~A}-\mathrm{C} 4-\mathrm{C} 5$ & 119.6 & $\mathrm{H} 22 \mathrm{~A}-\mathrm{C} 22-\mathrm{H} 22 \mathrm{~B}$ & 108.2 \\
\hline $\mathrm{N} 1-\mathrm{C} 5-\mathrm{N} 2$ & $115.03(17)$ & $\mathrm{H} 22 \mathrm{~A}-\mathrm{C} 22-\mathrm{C} 23$ & 109.7 \\
\hline $\mathrm{N} 1-\mathrm{C} 5-\mathrm{C} 4$ & $118.25(18)$ & $\mathrm{H} 22 \mathrm{~B}-\mathrm{C} 22-\mathrm{C} 23$ & 109.7 \\
\hline $\mathrm{N} 2-\mathrm{C} 5-\mathrm{C} 4$ & $126.56(17)$ & $\mathrm{O} 5-\mathrm{C} 23-\mathrm{C} 22$ & $107.88(14)$ \\
\hline $\mathrm{N} 2-\mathrm{C} 6-\mathrm{C} 7$ & $118.44(18)$ & $\mathrm{O} 5-\mathrm{C} 23-\mathrm{H} 23 \mathrm{~A}$ & 110.1 \\
\hline $\mathrm{N} 2-\mathrm{C} 6-\mathrm{C} 11$ & $125.59(18)$ & $\mathrm{O} 5-\mathrm{C} 23-\mathrm{H} 23 \mathrm{~B}$ & 110.1 \\
\hline $\mathrm{C} 7-\mathrm{C} 6-\mathrm{C} 11$ & $115.73(18)$ & $\mathrm{C} 22-\mathrm{C} 23-\mathrm{H} 23 \mathrm{~A}$ & 110.1 \\
\hline $\mathrm{C} 6-\mathrm{C} 7-\mathrm{H} 7 \mathrm{~A}$ & 118.9 & $\mathrm{C} 22-\mathrm{C} 23-\mathrm{H} 23 \mathrm{~B}$ & 110.1 \\
\hline $\mathrm{C} 6-\mathrm{C} 7-\mathrm{C} 8$ & $122.2(2)$ & $\mathrm{H} 23 \mathrm{~A}-\mathrm{C} 23-\mathrm{H} 23 \mathrm{~B}$ & 108.4 \\
\hline $\mathrm{H} 7 \mathrm{~A}-\mathrm{C} 7-\mathrm{C} 8$ & 118.9 & $\mathrm{O} 5-\mathrm{C} 24-\mathrm{C} 25$ & $124.30(16)$ \\
\hline $\mathrm{C} 7-\mathrm{C} 8-\mathrm{H} 8 \mathrm{~A}$ & 119.6 & $\mathrm{O} 5-\mathrm{C} 24-\mathrm{C} 29$ & $115.98(14)$ \\
\hline $\mathrm{C} 7-\mathrm{C} 8-\mathrm{C} 9$ & $120.8(2)$ & $\mathrm{C} 25-\mathrm{C} 24-\mathrm{C} 29$ & $119.73(16)$ \\
\hline $\mathrm{H} 8 \mathrm{~A}-\mathrm{C} 8-\mathrm{C} 9$ & 119.6 & $\mathrm{C} 24-\mathrm{C} 25-\mathrm{H} 25 \mathrm{~A}$ & 120.0 \\
\hline $\mathrm{C} 8-\mathrm{C} 9-\mathrm{H} 9 \mathrm{~A}$ & 120.7 & $\mathrm{C} 24-\mathrm{C} 25-\mathrm{C} 26$ & $119.94(18)$ \\
\hline $\mathrm{C} 8-\mathrm{C} 9-\mathrm{C} 10$ & $118.5(2)$ & $\mathrm{H} 25 \mathrm{~A}-\mathrm{C} 25-\mathrm{C} 26$ & 120.0 \\
\hline $\mathrm{H} 9 \mathrm{~A}-\mathrm{C} 9-\mathrm{C} 10$ & 120.7 & $\mathrm{C} 25-\mathrm{C} 26-\mathrm{H} 26 \mathrm{~A}$ & 119.7 \\
\hline $\mathrm{C} 9-\mathrm{C} 10-\mathrm{H} 10 \mathrm{~A}$ & 119.4 & $\mathrm{C} 25-\mathrm{C} 26-\mathrm{C} 27$ & $120.56(18)$ \\
\hline $\mathrm{C} 9-\mathrm{C} 10-\mathrm{C} 11$ & $121.1(2)$ & $\mathrm{H} 26 \mathrm{~A}-\mathrm{C} 26-\mathrm{C} 27$ & 119.7 \\
\hline $\mathrm{H} 10 \mathrm{~A}-\mathrm{C} 10-\mathrm{C} 11$ & 119.4 & $\mathrm{C} 26-\mathrm{C} 27-\mathrm{H} 27 \mathrm{~A}$ & 119.9 \\
\hline $\mathrm{C} 6-\mathrm{C} 11-\mathrm{C} 10$ & $121.6(2)$ & $\mathrm{C} 26-\mathrm{C} 27-\mathrm{C} 28$ & $120.25(18)$ \\
\hline $\mathrm{C} 6-\mathrm{C} 11-\mathrm{H} 11 \mathrm{~A}$ & 119.2 & $\mathrm{H} 27 \mathrm{~A}-\mathrm{C} 27-\mathrm{C} 28$ & 119.9 \\
\hline $\mathrm{C} 10-\mathrm{C} 11-\mathrm{H} 11 \mathrm{~A}$ & 119.2 & $\mathrm{C} 27-\mathrm{C} 28-\mathrm{H} 28 \mathrm{~A}$ & 120.0 \\
\hline $\mathrm{K}-\mathrm{O} 1-\mathrm{C} 12$ & $98.25(10)$ & $\mathrm{C} 27-\mathrm{C} 28-\mathrm{C} 29$ & $119.95(18)$ \\
\hline $\mathrm{K}-\mathrm{O} 1-\mathrm{C} 31$ & $97.49(9)$ & $\mathrm{H} 28 \mathrm{~A}-\mathrm{C} 28-\mathrm{C} 29$ & 120.0 \\
\hline $\mathrm{C} 12-\mathrm{O} 1-\mathrm{C} 31$ & $111.80(14)$ & $\mathrm{O} 6-\mathrm{C} 29-\mathrm{C} 24$ & $115.71(15)$ \\
\hline $\mathrm{K}-\mathrm{O} 2-\mathrm{C} 13$ & $113.71(10)$ & $\mathrm{O} 6-\mathrm{C} 29-\mathrm{C} 28$ & $124.77(16)$ \\
\hline $\mathrm{K}-\mathrm{O} 2-\mathrm{C} 14$ & $125.00(10)$ & $\mathrm{C} 24-\mathrm{C} 29-\mathrm{C} 28$ & $119.51(16)$ \\
\hline $\mathrm{C} 13-\mathrm{O} 2-\mathrm{C} 14$ & $116.73(14)$ & $\mathrm{O} 6-\mathrm{C} 30-\mathrm{H} 30 \mathrm{~A}$ & 110.0 \\
\hline $\mathrm{K}-\mathrm{O} 3-\mathrm{C} 19$ & $124.46(10)$ & $\mathrm{O} 6-\mathrm{C} 30-\mathrm{H} 30 \mathrm{~B}$ & 110.0 \\
\hline $\mathrm{K}-\mathrm{O} 3-\mathrm{C} 20$ & $116.65(10)$ & $\mathrm{O} 6-\mathrm{C} 30-\mathrm{C} 31$ & $108.36(14)$ \\
\hline $\mathrm{C} 19-\mathrm{O} 3-\mathrm{C} 20$ & $117.11(14)$ & $\mathrm{H} 30 \mathrm{~A}-\mathrm{C} 30-\mathrm{H} 30 \mathrm{~B}$ & 108.4 \\
\hline $\mathrm{K}-\mathrm{O} 4-\mathrm{C} 21$ & $110.08(10)$ & $\mathrm{H} 30 \mathrm{~A}-\mathrm{C} 30-\mathrm{C} 31$ & 110.0 \\
\hline $\mathrm{K}-\mathrm{O} 4-\mathrm{C} 22$ & $112.03(11)$ & $\mathrm{H} 30 \mathrm{~B}-\mathrm{C} 30-\mathrm{C} 31$ & 110.0 \\
\hline $\mathrm{C} 21-\mathrm{O} 4-\mathrm{C} 22$ & $113.97(16)$ & $\mathrm{O} 1-\mathrm{C} 31-\mathrm{C} 30$ & $109.54(16)$ \\
\hline $\mathrm{K}-\mathrm{O} 5-\mathrm{C} 23$ & $115.88(10)$ & $\mathrm{O} 1-\mathrm{C} 31-\mathrm{H} 31 \mathrm{~A}$ & 109.8 \\
\hline
\end{tabular}


$\mathrm{K}-\mathrm{O} 5-\mathrm{C} 24$

$\mathrm{C} 23-\mathrm{O} 5-\mathrm{C} 24$

$\mathrm{K}-\mathrm{O} 6-\mathrm{C} 29$

$\mathrm{K}-\mathrm{O} 6-\mathrm{C} 30$

$\mathrm{C} 29-\mathrm{O} 6-\mathrm{C} 30$

$\mathrm{N} 2-\mathrm{K}-\mathrm{N} 1-\mathrm{C} 1$

$\mathrm{N} 2-\mathrm{K}-\mathrm{N} 1-\mathrm{C} 5$

$\mathrm{O} 1-\mathrm{K}-\mathrm{N} 1-\mathrm{C} 1$

$\mathrm{O} 1-\mathrm{K}-\mathrm{N} 1-\mathrm{C} 5$

$\mathrm{O} 2-\mathrm{K}-\mathrm{N} 1-\mathrm{C} 1$

$\mathrm{O} 2-\mathrm{K}-\mathrm{N} 1-\mathrm{C} 5$

$\mathrm{O} 3-\mathrm{K}-\mathrm{N} 1-\mathrm{C} 1$

$\mathrm{O} 3-\mathrm{K}-\mathrm{N} 1-\mathrm{C} 5$

$\mathrm{O} 4-\mathrm{K}-\mathrm{N} 1-\mathrm{C} 1$

$\mathrm{O} 4-\mathrm{K}-\mathrm{N} 1-\mathrm{C} 5$

$\mathrm{O} 5-\mathrm{K}-\mathrm{N} 1-\mathrm{C} 1$

$\mathrm{O} 5-\mathrm{K}-\mathrm{N} 1-\mathrm{C} 5$

$\mathrm{O} 6-\mathrm{K}-\mathrm{N} 1-\mathrm{C} 1$

$\mathrm{O} 6-\mathrm{K}-\mathrm{N} 1-\mathrm{C} 5$

$\mathrm{N} 1-\mathrm{K}-\mathrm{N} 2-\mathrm{C} 5$

$\mathrm{N} 1-\mathrm{K}-\mathrm{N} 2-\mathrm{C} 6$

$\mathrm{O} 1-\mathrm{K}-\mathrm{N} 2-\mathrm{C} 5$

$\mathrm{O} 1-\mathrm{K}-\mathrm{N} 2-\mathrm{C} 6$

$\mathrm{O} 2-\mathrm{K}-\mathrm{N} 2-\mathrm{C} 5$

$\mathrm{O} 2-\mathrm{K}-\mathrm{N} 2-\mathrm{C} 6$

$\mathrm{O} 3-\mathrm{K}-\mathrm{N} 2-\mathrm{C} 5$

$\mathrm{O} 3-\mathrm{K}-\mathrm{N} 2-\mathrm{C} 6$

$\mathrm{O} 4-\mathrm{K}-\mathrm{N} 2-\mathrm{C} 5$

$\mathrm{O} 4-\mathrm{K}-\mathrm{N} 2-\mathrm{C} 6$

$\mathrm{O} 5-\mathrm{K}-\mathrm{N} 2-\mathrm{C} 5$

$\mathrm{O} 5-\mathrm{K}-\mathrm{N} 2-\mathrm{C} 6$

$\mathrm{O} 6-\mathrm{K}-\mathrm{N} 2-\mathrm{C} 5$

$\mathrm{O} 6-\mathrm{K}-\mathrm{N} 2-\mathrm{C} 6$

$\mathrm{K}-\mathrm{N} 1-\mathrm{C} 1-\mathrm{C} 2$

$\mathrm{C} 5-\mathrm{N} 1-\mathrm{C} 1-\mathrm{C} 2$

$\mathrm{N} 1-\mathrm{C} 1-\mathrm{C} 2-\mathrm{C} 3$

$\mathrm{C} 1-\mathrm{C} 2-\mathrm{C} 3-\mathrm{C} 4$

$\mathrm{C} 2-\mathrm{C} 3-\mathrm{C} 4-\mathrm{C} 5$

$\mathrm{K}-\mathrm{N} 2-\mathrm{C} 5-\mathrm{N} 1$

$\mathrm{K}-\mathrm{N} 2-\mathrm{C} 5-\mathrm{C} 4$

$\mathrm{C} 6-\mathrm{N} 2-\mathrm{C} 5-\mathrm{N} 1$

$\mathrm{C} 6-\mathrm{N} 2-\mathrm{C} 5-\mathrm{C} 4$

$\mathrm{K}-\mathrm{N} 1-\mathrm{C} 5-\mathrm{N} 2$

$\mathrm{K}-\mathrm{N} 1-\mathrm{C} 5-\mathrm{C} 4$

$\mathrm{C} 1-\mathrm{N} 1-\mathrm{C} 5-\mathrm{N} 2$

$\mathrm{C} 1-\mathrm{N} 1-\mathrm{C} 5-\mathrm{C} 4$

$\mathrm{C} 3-\mathrm{C} 4-\mathrm{C} 5-\mathrm{N} 1$
$123.80(10)$

$116.09(13)$

$125.40(10)$

$115.23(10)$

$117.00(13)$

$-167.6(2)$

$-3.90(9)$

$116.4(2)$

$-79.88(11)$

$57.6(2)$

$-138.70(10)$

$3.4(2)$

167.17 (10)

$-58.4(2)$

$105.39(10)$

$-106.9(2)$

$56.83(12)$

$-178.8(2)$

$-15.06(13)$

$4.02(10)$

$-170.6(2)$

117.14 (11)

$-57.47(18)$

$60.83(12)$

$-113.78(17)$

$-9.24(14)$

$176.14(15)$

$-70.45(11)$

114.94 (18)

$-131.65(11)$

$53.73(18)$

$175.32(11)$

$0.71(17)$

$161.11(16)$

$-0.5(3)$

$-0.5(3)$

1.9 (3)

$-2.5(3)$

$-6.85(17)$

$168.44(16)$

$168.74(17)$

$-16.0(3)$

$6.65(16)$

$-169.05(14)$

$175.69(17)$

$0.0(3)$

$1.5(3)$
$\mathrm{O} 1-\mathrm{C} 31-\mathrm{H} 31 \mathrm{~B}$

C $30-\mathrm{C} 31-\mathrm{H} 31 \mathrm{~A}$

$\mathrm{C} 30-\mathrm{C} 31-\mathrm{H} 31 \mathrm{~B}$

H31A-C $31-\mathrm{H} 31 \mathrm{~B}$

$\mathrm{N} 2-\mathrm{K}-\mathrm{O} 4-\mathrm{C} 21$

$\mathrm{N} 2-\mathrm{K}-\mathrm{O} 4-\mathrm{C} 22$

$\mathrm{O} 1-\mathrm{K}-\mathrm{O} 4-\mathrm{C} 21$

$\mathrm{O} 1-\mathrm{K}-\mathrm{O} 4-\mathrm{C} 22$

$\mathrm{O} 2-\mathrm{K}-\mathrm{O} 4-\mathrm{C} 21$

$\mathrm{O} 2-\mathrm{K}-\mathrm{O} 4-\mathrm{C} 22$

$\mathrm{O} 3-\mathrm{K}-\mathrm{O} 4-\mathrm{C} 21$

$\mathrm{O} 3-\mathrm{K}-\mathrm{O} 4-\mathrm{C} 22$

$\mathrm{O} 5-\mathrm{K}-\mathrm{O} 4-\mathrm{C} 21$

$\mathrm{O} 5-\mathrm{K}-\mathrm{O} 4-\mathrm{C} 22$

$\mathrm{O} 6-\mathrm{K}-\mathrm{O} 4-\mathrm{C} 21$

$\mathrm{O} 6-\mathrm{K}-\mathrm{O} 4-\mathrm{C} 22$

$\mathrm{N} 1-\mathrm{K}-\mathrm{O} 5-\mathrm{C} 23$

$\mathrm{N} 1-\mathrm{K}-\mathrm{O} 5-\mathrm{C} 24$

$\mathrm{N} 2-\mathrm{K}-\mathrm{O} 5-\mathrm{C} 23$

$\mathrm{N} 2-\mathrm{K}-\mathrm{O} 5-\mathrm{C} 24$

$\mathrm{O} 1-\mathrm{K}-\mathrm{O} 5-\mathrm{C} 23$

$\mathrm{O} 1-\mathrm{K}-\mathrm{O} 5-\mathrm{C} 24$

$\mathrm{O} 2-\mathrm{K}-\mathrm{O} 5-\mathrm{C} 23$

$\mathrm{O} 2-\mathrm{K}-\mathrm{O} 5-\mathrm{C} 24$

$\mathrm{O} 3-\mathrm{K}-\mathrm{O} 5-\mathrm{C} 23$

$\mathrm{O} 3-\mathrm{K}-\mathrm{O} 5-\mathrm{C} 24$

$\mathrm{O} 4-\mathrm{K}-\mathrm{O} 5-\mathrm{C} 23$

$\mathrm{O} 4-\mathrm{K}-\mathrm{O} 5-\mathrm{C} 24$

$\mathrm{O} 6-\mathrm{K}-\mathrm{O} 5-\mathrm{C} 23$

$\mathrm{O} 6-\mathrm{K}-\mathrm{O} 5-\mathrm{C} 24$

$\mathrm{N} 1-\mathrm{K}-\mathrm{O} 6-\mathrm{C} 29$

$\mathrm{N} 1-\mathrm{K}-\mathrm{O} 6-\mathrm{C} 30$

$\mathrm{N} 2-\mathrm{K}-\mathrm{O} 6-\mathrm{C} 29$

$\mathrm{N} 2-\mathrm{K}-\mathrm{O} 6-\mathrm{C} 30$

$\mathrm{O} 1-\mathrm{K}-\mathrm{O} 6-\mathrm{C} 29$

$\mathrm{O} 1-\mathrm{K}-\mathrm{O} 6-\mathrm{C} 30$

$\mathrm{O} 2-\mathrm{K}-\mathrm{O} 6-\mathrm{C} 29$

$\mathrm{O} 2-\mathrm{K}-\mathrm{O} 6-\mathrm{C} 30$

$\mathrm{O} 3-\mathrm{K}-\mathrm{O} 6-\mathrm{C} 29$

$\mathrm{O} 3-\mathrm{K}-\mathrm{O} 6-\mathrm{C} 30$

$\mathrm{O} 4-\mathrm{K}-\mathrm{O} 6-\mathrm{C} 29$

$\mathrm{O} 4-\mathrm{K}-\mathrm{O} 6-\mathrm{C} 30$

$\mathrm{O} 5-\mathrm{K}-\mathrm{O} 6-\mathrm{C} 29$

$\mathrm{O} 5-\mathrm{K}-\mathrm{O} 6-\mathrm{C} 30$

$\mathrm{K}-\mathrm{O} 1-\mathrm{C} 12-\mathrm{C} 13$

$\mathrm{C} 31-\mathrm{O} 1-\mathrm{C} 12-\mathrm{C} 13$
109.8

109.8

109.8

108.2

$109.38(13)$

$-122.70(12)$

$-98.33(18)$

$29.6(2)$

$-25.52(14)$

$102.39(12)$

$-28.33(12)$

99.59 (13)

$-159.41(14)$

$-31.49(11)$

-163.69 (12)

$-35.77(13)$

57.94 (13)

-97.97 (12)

98.49 (12)

$-57.42(12)$

$-166.00(11)$

38.09 (13)

$-97.83(12)$

$106.26(12)$

-45.08 (12)

159.01 (11)

-1.40 (11)

-157.31 (13)

173.87 (13)

17.96 (11)

96.36 (13)

$-65.53(13)$

88.05 (13)

-73.84 (12)

-174.04 (14)

24.06 (11)

$-150.19(12)$

47.92 (13)

$-87.12(13)$

110.98 (12)

-11.57 (13)

$-173.46(12)$

-16.12 (11)

-178.01 (13)

$-77.99(15)$

$-179.51(15)$ 


\begin{tabular}{|c|c|}
\hline $\mathrm{C} 3-\mathrm{C} 4-\mathrm{C} 5-\mathrm{N} 2$ & $-173.69(18)$ \\
\hline $\mathrm{K}-\mathrm{N} 2-\mathrm{C} 6-\mathrm{C} 7$ & $-41.0(3)$ \\
\hline $\mathrm{K}-\mathrm{N} 2-\mathrm{C} 6-\mathrm{C} 11$ & $133.05(18)$ \\
\hline $\mathrm{C} 5-\mathrm{N} 2-\mathrm{C} 6-\mathrm{C} 7$ & $145.31(18)$ \\
\hline $\mathrm{C} 5-\mathrm{N} 2-\mathrm{C} 6-\mathrm{C} 11$ & $-40.6(3)$ \\
\hline $\mathrm{N} 2-\mathrm{C} 6-\mathrm{C} 7-\mathrm{C} 8$ & $175.99(18)$ \\
\hline $\mathrm{C} 11-\mathrm{C} 6-\mathrm{C} 7-\mathrm{C} 8$ & $1.3(3)$ \\
\hline $\mathrm{C} 6-\mathrm{C} 7-\mathrm{C} 8-\mathrm{C} 9$ & $-0.8(3)$ \\
\hline $\mathrm{C} 7-\mathrm{C} 8-\mathrm{C} 9-\mathrm{C} 10$ & $-0.3(3)$ \\
\hline $\mathrm{C} 8-\mathrm{C} 9-\mathrm{C} 10-\mathrm{C} 11$ & $0.8(3)$ \\
\hline $\mathrm{C} 9-\mathrm{C} 10-\mathrm{C} 11-\mathrm{C} 6$ & $-0.2(3)$ \\
\hline $\mathrm{N} 2-\mathrm{C} 6-\mathrm{C} 11-\mathrm{C} 10$ & $-175.05(18)$ \\
\hline $\mathrm{C} 7-\mathrm{C} 6-\mathrm{C} 11-\mathrm{C} 10$ & $-0.8(3)$ \\
\hline $\mathrm{N} 1-\mathrm{K}-\mathrm{O} 1-\mathrm{C} 12$ & $-36.51(11)$ \\
\hline $\mathrm{N} 1-\mathrm{K}-\mathrm{O} 1-\mathrm{C} 31$ & $76.91(10)$ \\
\hline $\mathrm{N} 2-\mathrm{K}-\mathrm{O} 1-\mathrm{C} 12$ & $-82.45(11)$ \\
\hline $\mathrm{N} 2-\mathrm{K}-\mathrm{O} 1-\mathrm{C} 31$ & $30.97(10)$ \\
\hline $\mathrm{O} 2-\mathrm{K}-\mathrm{O} 1-\mathrm{C} 12$ & $43.30(10)$ \\
\hline $\mathrm{O} 2-\mathrm{K}-\mathrm{O} 1-\mathrm{C} 31$ & $156.72(11)$ \\
\hline $\mathrm{O} 3-\mathrm{K}-\mathrm{O} 1-\mathrm{C} 12$ & $61.69(11)$ \\
\hline $\mathrm{O} 3-\mathrm{K}-\mathrm{O} 1-\mathrm{C} 31$ & $175.11(10)$ \\
\hline $\mathrm{O} 4-\mathrm{K}-\mathrm{O} 1-\mathrm{C} 12$ & $124.64(16)$ \\
\hline $\mathrm{O} 4-\mathrm{K}-\mathrm{O} 1-\mathrm{C} 31$ & $-121.93(16)$ \\
\hline $\mathrm{O} 5-\mathrm{K}-\mathrm{O} 1-\mathrm{C} 12$ & $179.13(10)$ \\
\hline $\mathrm{O} 5-\mathrm{K}-\mathrm{O} 1-\mathrm{C} 31$ & $-67.44(10)$ \\
\hline $\mathrm{O} 6-\mathrm{K}-\mathrm{O} 1-\mathrm{C} 12$ & $-161.68(12)$ \\
\hline $\mathrm{O} 6-\mathrm{K}-\mathrm{O} 1-\mathrm{C} 31$ & $-48.26(10)$ \\
\hline $\mathrm{N} 1-\mathrm{K}-\mathrm{O} 2-\mathrm{C} 13$ & $96.99(12)$ \\
\hline $\mathrm{N} 1-\mathrm{K}-\mathrm{O} 2-\mathrm{C} 14$ & $-107.89(13)$ \\
\hline $\mathrm{N} 2-\mathrm{K}-\mathrm{O} 2-\mathrm{C} 13$ & $58.72(13)$ \\
\hline $\mathrm{N} 2-\mathrm{K}-\mathrm{O} 2-\mathrm{C} 14$ & $-146.15(12)$ \\
\hline $\mathrm{O} 1-\mathrm{K}-\mathrm{O} 2-\mathrm{C} 13$ & $-14.27(11)$ \\
\hline $\mathrm{O} 1-\mathrm{K}-\mathrm{O} 2-\mathrm{C} 14$ & $140.86(13)$ \\
\hline $\mathrm{O} 3-\mathrm{K}-\mathrm{O} 2-\mathrm{C} 13$ & $-173.03(13)$ \\
\hline $\mathrm{O} 3-\mathrm{K}-\mathrm{O} 2-\mathrm{C} 14$ & $-17.91(11)$ \\
\hline $\mathrm{O} 4-\mathrm{K}-\mathrm{O} 2-\mathrm{C} 13$ & $-176.09(12)$ \\
\hline $\mathrm{O} 4-\mathrm{K}-\mathrm{O} 2-\mathrm{C} 14$ & $-20.96(13)$ \\
\hline $\mathrm{O} 5-\mathrm{K}-\mathrm{O} 2-\mathrm{C} 13$ & $-102.07(13)$ \\
\hline $\mathrm{O} 5-\mathrm{K}-\mathrm{O} 2-\mathrm{C} 14$ & $53.06(14)$ \\
\hline $\mathrm{O} 6-\mathrm{K}-\mathrm{O} 2-\mathrm{C} 13$ & $-37.77(13)$ \\
\hline $\mathrm{O} 6-\mathrm{K}-\mathrm{O} 2-\mathrm{C} 14$ & $117.36(12)$ \\
\hline $\mathrm{N} 1-\mathrm{K}-\mathrm{O} 3-\mathrm{C} 19$ & $109.73(13)$ \\
\hline $\mathrm{N} 1-\mathrm{K}-\mathrm{O} 3-\mathrm{C} 20$ & $-85.99(12)$ \\
\hline $\mathrm{N} 2-\mathrm{K}-\mathrm{O} 3-\mathrm{C} 19$ & $119.50(13)$ \\
\hline $\mathrm{N} 2-\mathrm{K}-\mathrm{O} 3-\mathrm{C} 20$ & $-76.22(14)$ \\
\hline $\mathrm{O} 1-\mathrm{K}-\mathrm{O} 3-\mathrm{C} 19$ & $-0.69(13)$ \\
\hline $\mathrm{O} 1-\mathrm{K}-\mathrm{O} 3-\mathrm{C} 20$ & $163.59(12)$ \\
\hline $\mathrm{O} 2-\mathrm{K}-\mathrm{O} 3-\mathrm{C} 19$ & $19.09(12)$ \\
\hline
\end{tabular}

\begin{tabular}{|c|c|}
\hline $\mathrm{K}-\mathrm{O} 2-\mathrm{C} 13-\mathrm{C} 12$ & $-17.02(18)$ \\
\hline $\mathrm{C} 14-\mathrm{O} 2-\mathrm{C} 13-\mathrm{C} 12$ & $-174.33(15)$ \\
\hline $\mathrm{O} 1-\mathrm{C} 12-\mathrm{C} 13-\mathrm{O} 2$ & $68.2(2)$ \\
\hline $\mathrm{K}-\mathrm{O} 2-\mathrm{C} 14-\mathrm{C} 15$ & $-163.90(14)$ \\
\hline $\mathrm{K}-\mathrm{O} 2-\mathrm{C} 14-\mathrm{C} 19$ & $16.4(2)$ \\
\hline $\mathrm{C} 13-\mathrm{O} 2-\mathrm{C} 14-\mathrm{C} 15$ & $-9.4(3)$ \\
\hline $\mathrm{C} 13-\mathrm{O} 2-\mathrm{C} 14-\mathrm{C} 19$ & $170.87(15)$ \\
\hline $\mathrm{O} 2-\mathrm{C} 14-\mathrm{C} 15-\mathrm{C} 16$ & $178.94(17)$ \\
\hline $\mathrm{C} 19-\mathrm{C} 14-\mathrm{C} 15-\mathrm{C} 16$ & $-1.4(3)$ \\
\hline $\mathrm{C} 14-\mathrm{C} 15-\mathrm{C} 16-\mathrm{C} 17$ & $0.1(3)$ \\
\hline $\mathrm{C} 15-\mathrm{C} 16-\mathrm{C} 17-\mathrm{C} 18$ & $0.8(3)$ \\
\hline $\mathrm{C} 16-\mathrm{C} 17-\mathrm{C} 18-\mathrm{C} 19$ & $-0.3(3)$ \\
\hline $\mathrm{C} 17-\mathrm{C} 18-\mathrm{C} 19-\mathrm{O} 3$ & $178.15(16)$ \\
\hline $\mathrm{C} 17-\mathrm{C} 18-\mathrm{C} 19-\mathrm{C} 14$ & $-1.0(3)$ \\
\hline $\mathrm{K}-\mathrm{O} 3-\mathrm{C} 19-\mathrm{C} 14$ & $-20.1(2)$ \\
\hline $\mathrm{K}-\mathrm{O} 3-\mathrm{C} 19-\mathrm{C} 18$ & $160.64(13)$ \\
\hline $\mathrm{C} 20-\mathrm{O} 3-\mathrm{C} 19-\mathrm{C} 14$ & $175.63(15)$ \\
\hline $\mathrm{C} 20-\mathrm{O} 3-\mathrm{C} 19-\mathrm{C} 18$ & $-3.6(2)$ \\
\hline $\mathrm{O} 2-\mathrm{C} 14-\mathrm{C} 19-\mathrm{O} 3$ & $2.3(2)$ \\
\hline $\mathrm{O} 2-\mathrm{C} 14-\mathrm{C} 19-\mathrm{C} 18$ & $-178.44(15)$ \\
\hline $\mathrm{C} 15-\mathrm{C} 14-\mathrm{C} 19-\mathrm{O} 3$ & $-177.40(16)$ \\
\hline $\mathrm{C} 15-\mathrm{C} 14-\mathrm{C} 19-\mathrm{C} 18$ & $1.9(3)$ \\
\hline $\mathrm{K}-\mathrm{O} 3-\mathrm{C} 20-\mathrm{C} 21$ & $25.82(19)$ \\
\hline $\mathrm{C} 19-\mathrm{O} 3-\mathrm{C} 20-\mathrm{C} 21$ & $-168.71(16)$ \\
\hline $\mathrm{K}-\mathrm{O} 4-\mathrm{C} 21-\mathrm{C} 20$ & $59.5(2)$ \\
\hline $\mathrm{C} 22-\mathrm{O} 4-\mathrm{C} 21-\mathrm{C} 20$ & $-67.4(2)$ \\
\hline $\mathrm{O} 3-\mathrm{C} 20-\mathrm{C} 21-\mathrm{O} 4$ & $-58.0(2)$ \\
\hline $\mathrm{K}-\mathrm{O} 4-\mathrm{C} 22-\mathrm{C} 23$ & $63.26(17)$ \\
\hline $\mathrm{C} 21-\mathrm{O} 4-\mathrm{C} 22-\mathrm{C} 23$ & $-170.92(14)$ \\
\hline $\mathrm{K}-\mathrm{O} 5-\mathrm{C} 23-\mathrm{C} 22$ & $30.54(19)$ \\
\hline $\mathrm{C} 24-\mathrm{O} 5-\mathrm{C} 23-\mathrm{C} 22$ & $-171.65(15)$ \\
\hline $\mathrm{O} 4-\mathrm{C} 22-\mathrm{C} 23-\mathrm{O} 5$ & $-62.1(2)$ \\
\hline $\mathrm{K}-\mathrm{O} 5-\mathrm{C} 24-\mathrm{C} 25$ & $160.33(14)$ \\
\hline $\mathrm{K}-\mathrm{O} 5-\mathrm{C} 24-\mathrm{C} 29$ & $-19.7(2)$ \\
\hline $\mathrm{C} 23-\mathrm{O} 5-\mathrm{C} 24-\mathrm{C} 25$ & $4.5(2)$ \\
\hline $\mathrm{C} 23-\mathrm{O} 5-\mathrm{C} 24-\mathrm{C} 29$ & $-175.53(16)$ \\
\hline $\mathrm{O} 5-\mathrm{C} 24-\mathrm{C} 25-\mathrm{C} 26$ & $178.66(17)$ \\
\hline $\mathrm{C} 29-\mathrm{C} 24-\mathrm{C} 25-\mathrm{C} 26$ & $-1.3(3)$ \\
\hline $\mathrm{C} 24-\mathrm{C} 25-\mathrm{C} 26-\mathrm{C} 27$ & $-0.6(3)$ \\
\hline $\mathrm{C} 25-\mathrm{C} 26-\mathrm{C} 27-\mathrm{C} 28$ & $1.0(3)$ \\
\hline $\mathrm{C} 26-\mathrm{C} 27-\mathrm{C} 28-\mathrm{C} 29$ & $0.6(3)$ \\
\hline $\mathrm{K}-\mathrm{O} 6-\mathrm{C} 29-\mathrm{C} 24$ & $13.9(2)$ \\
\hline $\mathrm{K}-\mathrm{O} 6-\mathrm{C} 29-\mathrm{C} 28$ & $-165.13(13)$ \\
\hline $\mathrm{C} 30-\mathrm{O} 6-\mathrm{C} 29-\mathrm{C} 24$ & $175.55(16)$ \\
\hline $\mathrm{C} 30-\mathrm{O} 6-\mathrm{C} 29-\mathrm{C} 28$ & $-3.5(3)$ \\
\hline $\mathrm{C} 27-\mathrm{C} 28-\mathrm{C} 29-\mathrm{O} 6$ & $176.51(17)$ \\
\hline $\mathrm{C} 27-\mathrm{C} 28-\mathrm{C} 29-\mathrm{C} 24$ & $-2.5(3)$ \\
\hline $\mathrm{O} 5-\mathrm{C} 24-\mathrm{C} 29-\mathrm{O} 6$ & $3.8(2)$ \\
\hline
\end{tabular}




$\begin{array}{llll}\mathrm{O} 2-\mathrm{K}-\mathrm{O} 3-\mathrm{C} 20 & -176.63(13) & \mathrm{O} 5-\mathrm{C} 24-\mathrm{C} 29-\mathrm{C} 28 & -177.12(15) \\ \mathrm{O} 4-\mathrm{K}-\mathrm{O} 3-\mathrm{C} 19 & -164.03(13) & \mathrm{C} 25-\mathrm{C} 24-\mathrm{C} 29-\mathrm{O} 6 & -176.23(16) \\ \mathrm{O} 4-\mathrm{K}-\mathrm{O} 3-\mathrm{C} 20 & 0.26(12) & \mathrm{C} 25-\mathrm{C} 24-\mathrm{C} 29-\mathrm{C} 28 & 2.9(3) \\ \mathrm{O} 5-\mathrm{K}-\mathrm{O} 3-\mathrm{C} 19 & -121.06(12) & \mathrm{K}-\mathrm{O} 6-\mathrm{C} 30-\mathrm{C} 31 & 4.57(19) \\ \mathrm{O} 5-\mathrm{K}-\mathrm{O} 3-\mathrm{C} 20 & 43.22(13) & \mathrm{C} 29-\mathrm{O} 6-\mathrm{C} 30-\mathrm{C} 31 & -158.91(15) \\ \mathrm{O} 6-\mathrm{K}-\mathrm{O} 3-\mathrm{C} 19 & -67.58(14) & \mathrm{K}-\mathrm{O} 1-\mathrm{C} 31-\mathrm{C} 30 & 78.67(14) \\ \mathrm{O} 6-\mathrm{K}-\mathrm{O} 3-\mathrm{C} 20 & 96.71(13) & \mathrm{C} 12-\mathrm{O} 1-\mathrm{C} 31-\mathrm{C} 30 & -179.30(15) \\ \mathrm{N} 1-\mathrm{K}-\mathrm{O} 4-\mathrm{C} 21 & 63.83(13) & \mathrm{O} 6-\mathrm{C} 30-\mathrm{C} 31-\mathrm{O} 1 & -59.1(2) \\ \mathrm{N} 1-\mathrm{K}-\mathrm{O} 4-\mathrm{C} 22 & -168.25(12) & & \end{array}$

